\title{
Swarm based Intelligent Feature Optimization Technique for ECG based Biometric Human Recognition
}

\author{
Kiran Kumar Patro, M. Jayamanmadha Rao, P.Rajesh Kumar
}

\begin{abstract}
Recently, rapid growth in network technology and communication leads to widening human activities, so that a strong identification system is necessary. This work aims to build an accurate identification technology based on unique physiological characteristics of ECG. The Biometric recognition of ECG primarily depends on the quality of its features. Feature extraction is performed on parameters of a cardiac cycle based on the fiducial approach and large data sets of features have been extracted. The extracted dataset contains irrelevant, correlated and over-fitted features, which misleads the biometric system performance so that an effective feature optimization is needed to sort out those features to avoid redundancy in the data. In this paper, a novel swarm based intelligent feature optimization method; Particle Swarm Optimization (PSO) is used to generate feature subset based on a fitness function with joint entropy. The dataset from optimization phase are fed to classifiers such as ANN, K-NN and $S V M$ for recognition. The proposed approach is tested with available open source MIT-BIH ECG ID database. Finally, a comparison is made with and without feature optimization in which PSO with KNN shows recognition accuracy of $97.8931 \%$.
\end{abstract}

Index Terms: Biometric, Electrocardiogram (ECG), Entropy, SVM, Swarm.

\section{INTRODUCTION}

Generally, biometrics are related to living beings. A biometric recognition is similar to a pattern recognition system that can recognize a person in terms of specific physiological or behavioral features [1]. There are different types of conventional biometric traits such as fingerprint, iris, voice and face which are playing different roles on their recognition. These identification schemes have the limitation of falsification and may not meet the higher security demands. Now a days, using biomedical signals in the field of security becomes popular. Many studies have been proposed on Electrocardiogram for biometric personal identification systems [2-5].

Compared to traditional biometric traits, ECG provides more secure biometric recognition with liveness of the subject. A perfect biometric should include the characteristics of universal, unique, easily measured, and

Revised Version Manuscript Received on Jun 20, 2019.

Kiran Kumar Patro, ECE, Aditya Institute of Technology and Managemant, Tekkali, Andhra Pradesh, India.

M.Jayamanmadha Rao, ECE, Aditya Institute of Technology and Managemant, Tekkali, Andhra Pradesh, India..

P.Rajesh Kumar, ECE, Andhra University, Visakhapatnam, Andhra Pradesh, India. permanent. ECG satisfies most of the conditions for perfect biometric. There are some experimental discussions that support ECG as a human biometric characteristic ECG is a universal and it is a significant indication of life probably ECG is almost unique and depends on the morphology of heart and body anatomic features of each individual. Fig.1 shows the characteristic points of Electrocardiogram beat.

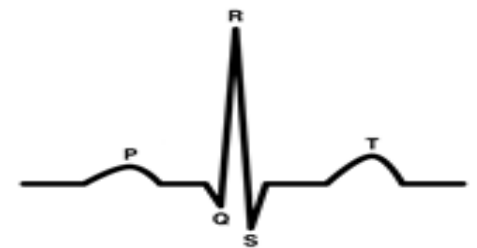

Fig.1 ECG pulse with P-QRS-T Points

Further, ECG is measured quite convenient with Lead-I, which is preferred such that it can recorded easily and not sensitive to small variations. Finally, ECG is permanent during a certain time period and it does not change suddenly over time.

In the literature, various techniques for heart beat classification procedures have been proposed for ECG as biometric application. Tilendra et al. [6] suggested biometric recognition using ECG signals with normalized cross correlation metric. Sairul safie et al. [7] proposed to extract bipolar active features and the authentication performance was computed with and without classifier of Linear Discriminant Analysis (LDA). Md. Khayrul Bashar et al. [8] proposed ECG biometric recognition using mulscale descriptors. The presented new method which extracts multi scale geometrical features from ECG signals.

Abhijit Sarkar et al. [9] proposed a new methodology in ECG biometrics, in that a dynamic system model is presented to improve enrollment task of ECG pulses and utilized sum of Gaussian representation methods to detect different parameters at fiducial points. Yue Zhang et al. [10] introduced a hierarchical classifier for template matching in ECG based recognition. In view of physiological variability, Fatema-tuz-Zohra Iqbal et al. [11] proposed a comprehensive analysis of QRS complex and also introduced cardioid graph based biometric authentication system. In this system features were extracted from selected QRS complex graph and recognition performed using MLPNN classifier.

The proposed work mainly concentrated on extracting features and selection of optimized features from ECG signal. In this work, systematic approach for extracting time 
domain features are adopted. Moreover, swarm based technique is proposed for intelligent selection of features for classification.

The paper is organized into 5 sections: Section. 1 presents a small introduction to different conventional biometric traits. Methodology for ECG based biometric recognition system discussed in section 2. In section.3 swarm based feature selection technique is proposed. Further, section 4 and 5 illustrates experimental results and conclusion.

\section{RECOGNITION SYSTEM METHODOLOGY}

ECG based biometric system uses a classical scheme, which includes ECG data acquisition, pre-processing, P-QRS-T fiducial detection, effective feature extraction, feature optimization and classification and identification as shown in Fig. 2.

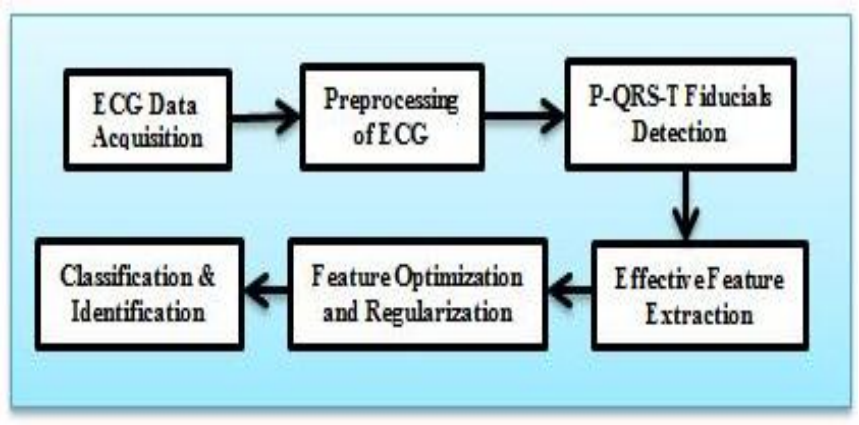

Fig.2. Recognition system synthesis

Out of all the stages, effective feature extraction and optimized feature selection stages are gained lot of importance in performance of biometric system. In many studies, non fiducial based features (Wavelet transform) are utilized in ECG based recognition [12]. In recent works, Wan and Yao [13] extracted 256 biorthogonal wavelet coefficients from ECG beat for identification. Belgacem et al. [14] introduced different levels $(\mathrm{db} 3 / \mathrm{db} 5)$ of DWT coefficients as features for recognition. In this work, a time-frequency analysis is used for finding P-QRS-T characteristic points [15]. After detection, it is noted that durations of QRS, ST and QT are varied while changing heart rate therefore finest PQRST beats were selected for feature extraction. Each beat was fragmented starting from $P$ peak to $T$ peak and there by selecting 6 best fragments by their least difference from the mean of Euclidian distance of the peaks shown in Fig.3 [16], [17].

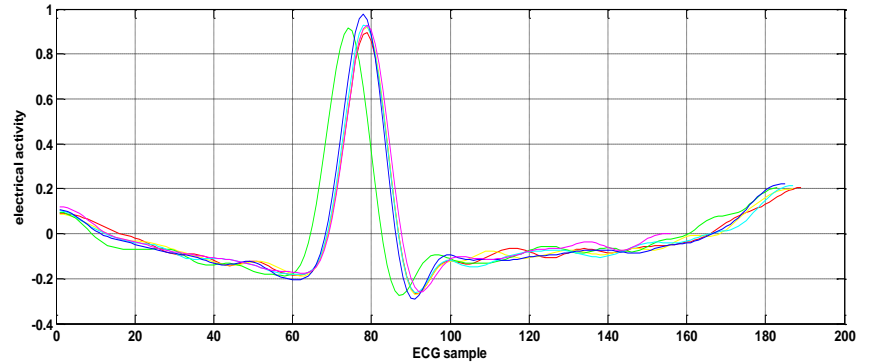

Fig.3. ECG beat of best six PQRST fragment

Still, the independent PQRST fragments are not suitable to extract data from ECG due to non-uniformity. To overcome this, the position of R-peak is translated to new standard point (origin) and such that the positions of all other characteristic points $(\mathrm{P}, \mathrm{Q}, \mathrm{S}$ and $\mathrm{T})$ are also shifted. This brings the uniformity in the data shown in Fig. 4.

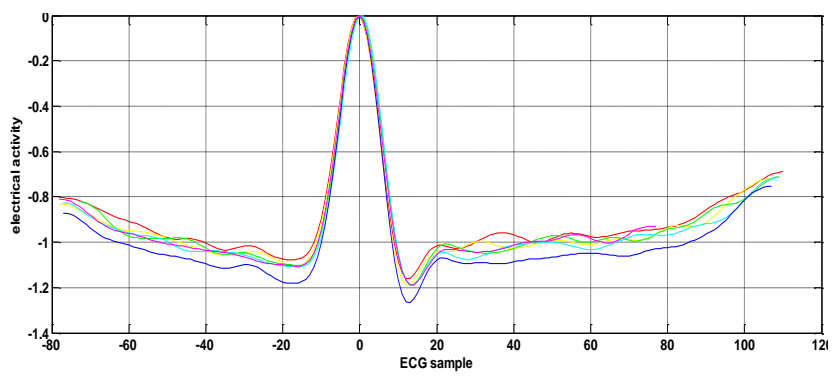

Fig.4. Best six PQRST fragments with the position Normalized

In data extraction, all the time intervals and amplitudes were calculated with respect to R-peak position as origin. Based on fiducials different features are extracted shown in Table 1.

\section{FEATURE OPTIMIZATION}

Optimization is the process of selecting appropriate element from set of available choices. The selection of best element is based on the strategy of objective function. Optimized feature selection methods reduce feature space, thereby improving system accuracy and reducing computation time [18]. Large Feature set $(\mathrm{N}=72)$ may contain irrelevant, correlated and noisy features, which might result in misleading the classification technique Those features should be sorted out from dataset [19]. Feature optimization generates smaller feature subset from a superset of extracted features. Generated subset goes to evaluation based on a specific strategy, this will repeat until stopping criteria is met.

\section{A. Particle Swarm Optimization (PSO)}

PSO is used in resolving varies pattern recognition problems. Unlike evolutionary algorithms, PSO is a swarm based intelligent optimization technique which shows computational simplicity and time. PSO is inspired by swarm intelligence perceived in fish schooling and bird flocking [20]. This method is depends on assigning data between swarm members or particles. A cost function is assigned to each particle to compute fitness. The individual particles change their direction and velocity towards the best cost particle. The process is repeated until to get optimum results [21].

The Working mechanism of PSO follows below mentioned steps:

1. Generate randomly a swarm of size $\mathrm{S}(\mathrm{N}=72)$

2. Initialize random Position $(\mathrm{P})$ and Velocity $(\mathrm{V})$ of each particle.

3. Calculate fitness (Objective function) of each particle and evaluate its Position based on fitness.

4. If fitness $(\mathrm{P})$ is better than fitness of $\left(\mathrm{P}_{\text {best }}\right)$ then update $\mathrm{P}_{\text {best }}=\mathrm{P}$.

5. Out of all $P_{\text {best }}$ of particles set $G_{\text {best }}$.

6. Update particle Position (P) and Velocity (V) and repeat from step-2 until stopping condition is met 
Table 1. Extracted features with the position of ' $R$ ' as origin

\begin{tabular}{|c|c|c|c|c|}
\hline Features & \multicolumn{4}{|c|}{ Features Description } \\
\hline \multirow{3}{*}{ I. Time Features } & 1.Px & 2.Qx & 3.Sx & 4.Tx \\
\hline & 5.PQ & 6.PT & 7.QS & 8.QT \\
\hline & 9.ST & 10.PS & 11.PT/QS & 12.QT/QS \\
\hline \multirow{6}{*}{ II. Amplitude Features } & 13.Py & 14.Qy & 15.Sy & 16.Ty \\
\hline & 17.PQ & 18.QR & 19.RS & 20.ST \\
\hline & 21.QS & 22.PS & 23.PT & 24.QT \\
\hline & 25.ST/QS & 26.RS/QR & 27.PQ/QS & 28.PQ/QT \\
\hline & 29.PQ/PS & 30.PQ/QR & 31.PQ/RS & 32.RS/QS \\
\hline & 33.RS/QT & 34.ST/PQ & 35.ST/QT & \\
\hline \multirow[t]{2}{*}{ III. Distance Features } & 36.PQ & 37.QR & $38 . \mathrm{RS}$ & 39.ST \\
\hline & 40.QS & 41.PR & 42. ST/QS & 43.RS/QR \\
\hline \multirow{3}{*}{ IV.Slope Features } & 44.PQ & $45 . \mathrm{QR}$ & 46.RS & 47.ST \\
\hline & 48.QS & 49.PT & 50.PS & 51.QT \\
\hline & 52.PR & & & \\
\hline \multirow[b]{2}{*}{ V. Angle Features } & 53.PQR & 54.QRS & 55.RST & 56.RQS \\
\hline & 57.RSQ & 58.RTS & & \\
\hline \multirow{4}{*}{$\begin{array}{l}\text { VI. Miscellaneous } \\
\text { Features }\end{array}$} & 59.QRS area & 60.QRSarea/RS^2 & 61.(R/S)angl & 62.R angl/QStime \\
\hline & 63.S angl/QTtime & 64.S angl/PQ dis & 65.(R/Q) angl & 66.(R/T)angl \\
\hline & 67.(Q/T) angl & 68.QRSarea/QRamp & 69.QRS perimeter & 70.QRS in radius \\
\hline & 71.QRSxcentroid & 72.QRSycentroid & & \\
\hline
\end{tabular}

\section{B. Fitness Function}

The fitness function or objective function is used to estimate quality of population based on different strategies. It will search for optimal values for the feature selection. The appropriate fitness function used in this work is based on Shannon entropy function [22].

$$
\begin{gathered}
W=\text { mean }_{-} I x y-C * \text { mean_Ixx } \\
\text { mean }_{-} I x y=\frac{\text { mean }_{-} I x y}{\text { feat_numb }} \cdot \\
\text { mean_Ixx }=\frac{\text { mean_Ixx-feat_numb }}{\text { feat_numb }{ }^{2}-f e a t_{-} n u m b}
\end{gathered}
$$

Where, mean_Ixy is the Mutual information calculated between the features and output. This function removes relevant data between the features and output. mean_Ixx is the Mutual Information of all the features. This function eliminates redundant data between the features. $\mathrm{C}$ is an arbitrary constant.

Features are selected depending on the maximum fitness value in that iteration within the boundary limits defined by W_max and W_min values. mean_Ixx and mean_Ixy calculates their mutual information depending upon the entropy value of feature. The Fitness function calculates the fitness of every feature that is fed to optimization phase. It is designed in such a way that it eliminates the correlated, random and over-fitted features. The fitness curve shown in Fig.5 represents the target fitness level achieved at successive iterations by the set of selected features out of available features. It indicates a graph of several iterations versus the best cost of the corresponding selected features. Finally, at 30th iteration 30 best features are generated as shown in Table 2.

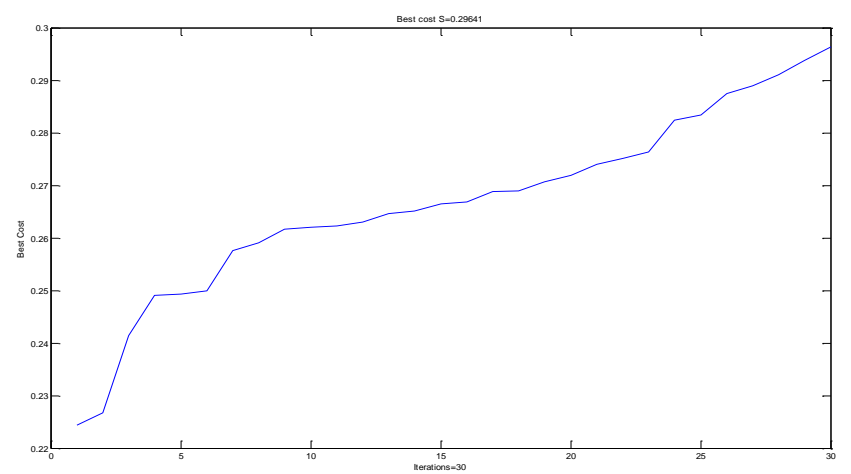

Fig.5 Fitness Curve of PSO 
Table 2. Set of optimized features reproduced at $30^{\text {th }}$ iteration of PSO

\begin{tabular}{cclc}
\hline S.No & $\begin{array}{c}\text { Selected } \\
\text { Feature no. }\end{array}$ & $\begin{array}{l}\text { Feature } \\
\text { Name }\end{array}$ & $\begin{array}{c}\text { Fitness } \\
\text { Value }\end{array}$ \\
\hline $\mathbf{1}$ & 8 & QTtime & 0.2243 \\
$\mathbf{2}$ & 20 & Stamp & 0.2268 \\
$\mathbf{3}$ & 59 & QRSarea & 0.2414 \\
$\mathbf{4}$ & 40 & QSdis & 0.2490 \\
$\mathbf{5}$ & 14 & Qy & 0.2492 \\
$\mathbf{6}$ & 30 & (PQ/QR)amp & 0.2499 \\
$\mathbf{7}$ & 38 & RSdis & 0.2575 \\
$\mathbf{8}$ & 46 & RSslope & 0.2591 \\
$\mathbf{9}$ & 24 & QTamp & 0.2616 \\
$\mathbf{1 0}$ & 9 & STtime & 0.2619 \\
$\mathbf{1 1}$ & 28 & (PQ/QT)amp & 0.2622 \\
$\mathbf{1 2}$ & 12 & (QT/QS)time & 0.2629 \\
$\mathbf{1 3}$ & 1 & Px & 0.2646 \\
$\mathbf{1 4}$ & 10 & PStime & 0.2651 \\
$\mathbf{1 5}$ & 2 & Qx & 0.2664 \\
$\mathbf{1 6}$ & 13 & Py & 0.2668 \\
$\mathbf{1 7}$ & 6 & PTtime & 0.2688 \\
$\mathbf{1 8}$ & 45 & QRslope & 0.2689 \\
$\mathbf{1 9}$ & 3 & Sx & 0.2706 \\
$\mathbf{2 0}$ & 26 & RS/QRamp & 0.2719 \\
$\mathbf{2 1}$ & 34 & (ST/PQ)amp & 0.2739 \\
$\mathbf{2 2}$ & 15 & Sy & 0.2750 \\
$\mathbf{2 3}$ & 66 & R/Tangle & 0.2763 \\
$\mathbf{2 4}$ & 4 & Tx & 0.2824 \\
$\mathbf{2 5}$ & 31 & (PQ/RS)amp & 0.2833 \\
$\mathbf{2 6}$ & 15 & Ty & 0.2874 \\
$\mathbf{2 7}$ & 33 & (RS/QT)amp & 0.2889 \\
$\mathbf{2 8}$ & 17 & PQamp & 0.2910 \\
$\mathbf{2 9}$ & 11 & (PT/QS)time & 0.2938 \\
$\mathbf{3 0}$ & 36 & PQdis & 0.2964 \\
\hline & & & \\
& & &
\end{tabular}

\section{EXPERIMENTAL RESULTS}

In this research, performance of the proposed method is evaluated by doing experiments on public available MIT-BIH ECG ID database from Physionet [23]. Five signals of ECG with different heart rates of each individual have been considered. Out of which 2 signals are used for training and remaining records are used for testing the system.

Table 3. Confusion Matrix generated for 20 subjects using PSO+ANN (\%)

\begin{tabular}{cccccccccccccccccccccc}
\hline & $\mathbf{A}$ & $\mathbf{B}$ & $\mathbf{C}$ & $\mathbf{D}$ & $\mathbf{E}$ & $\mathbf{F}$ & $\mathbf{G}$ & $\mathbf{H}$ & $\mathbf{I}$ & $\mathbf{J}$ & $\mathbf{K}$ & $\mathbf{L}$ & $\mathbf{M}$ & $\mathbf{N}$ & $\mathbf{O}$ & $\mathbf{P}$ & $\mathbf{Q}$ & $\mathbf{R}$ & $\mathbf{S}$ & $\mathbf{T}$ \\
\hline $\mathbf{A}$ & $\mathbf{1 0 0}$ & 0 & 0 & 0 & 0 & 0 & 0 & 0 & 0 & 0 & 0 & 0 & 0 & 33.3 & 0 & 0 & 0 & 0 & 0 & 0 \\
$\mathbf{B}$ & 0 & $\mathbf{1 0 0}$ & 0 & 0 & 0 & 0 & 66.7 & 0 & 0 & 0 & 66.7 & 0 & 0 & 0 & 0 & 0 & 0 & 0 & 0 & 0 & 0 \\
$\mathbf{C}$ & 0 & 0 & $\mathbf{1 0 0}$ & 0 & 0 & 0 & 0 & 0 & 0 & 0 & 0 & 0 & 0 & 0 & 0 & 0 & 0 & 0 & 0 & 0 \\
$\mathbf{D}$ & 0 & 0 & 0 & $\mathbf{1 0 0}$ & 0 & 0 & 0 & 0 & 0 & 0 & 0 & 0 & 0 & 0 & 0 & 0 & 0 & 0 & 0 & 0 & 0 \\
$\mathbf{E}$ & 0 & 0 & 0 & 0 & $\mathbf{6 6 . 7}$ & 0 & 0 & 0 & 0 & 0 & 0 & 0 & 0 & 0 & 0 & 0 & 0 & 0 & 0 & 0 & 0 \\
$\mathbf{F}$ & 0 & 0 & 0 & 0 & 0 & $\mathbf{1 0 0}$ & 0 & 0 & 0 & 0 & 0 & 0 & 0 & 0 & 0 & 0 & 0 & 0 & 0 & 0 & 0 \\
$\mathbf{G}$ & 0 & 0 & 0 & 0 & 0 & 0 & $\mathbf{3 3 . 3}$ & 0 & 0 & 0 & 0 & 0 & 0 & 0 & 0 & 0 & 0 & 0 & 0 & 0 & 0 \\
$\mathbf{H}$ & 0 & 0 & 0 & 0 & 0 & 0 & 0 & $\mathbf{1 0 0}$ & 0 & 0 & 0 & 0 & 0 & 0 & 0 & 0 & 0 & 0 & 0 & 0 \\
$\mathbf{I}$ & 0 & 0 & 0 & 0 & 0 & 0 & 0 & 0 & $\mathbf{1 0 0}$ & 0 & 0 & 0 & 0 & 0 & 0 & 0 & 0 & 0 & 0 & 0 \\
$\mathbf{J}$ & 0 & 0 & 0 & 0 & 0 & 0 & 0 & 0 & 0 & $\mathbf{6 6 . 7}$ & 0 & 0 & 0 & 0 & 0 & 0 & 0 & 0 & 0 & 0 \\
$\mathbf{K}$ & 0 & 0 & 0 & 0 & 0 & 0 & 0 & 0 & 0 & 0 & $\mathbf{3 3 . 3}$ & 0 & 0 & 0 & 0 & 0 & 0 & 0 & 0 & 0 \\
$\mathbf{L}$ & 0 & 0 & 0 & 0 & 0 & 0 & 0 & 0 & 0 & 0 & 0 & $\mathbf{1 0 0}$ & 0 & 0 & 0 & 0 & 0 & 0 & 0 & 0 \\
$\mathbf{M}$ & 0 & 0 & 0 & 0 & 0 & 0 & 0 & 0 & 0 & 33.3 & 0 & 0 & $\mathbf{1 0 0}$ & 0 & 0 & 0 & 0 & 33.3 & 0 & 0 \\
$\mathbf{N}$ & 2 & 0 & 0 & 0 & 0 & 0 & 0 & 0 & 0 & 0 & 0 & 0 & 0 & $\mathbf{6 6 . 7}$ & 0 & 0 & 0 & 0 & 0 & 0 \\
$\mathbf{O}$ & 0 & 0 & 0 & 0 & 0 & 0 & 0 & 0 & 0 & 0 & 0 & 0 & 0 & 0 & $\mathbf{1 0 0}$ & 0 & 0 & 0 & 0 & 0 \\
$\mathbf{P}$ & 0 & 0 & 0 & 0 & 0 & 0 & 0 & 0 & 0 & 0 & 0 & 0 & 0 & 0 & 0 & $\mathbf{1 0 0}$ & 0 & 0 & 0 & 0 \\
$\mathbf{Q}$ & 0 & 0 & 0 & 0 & 0 & 0 & 0 & 0 & 0 & 0 & 0 & 0 & 0 & 0 & 0 & 0 & $\mathbf{1 0 0}$ & 0 & 0 & 33.3
\end{tabular}




\begin{tabular}{|c|c|c|c|c|c|c|c|c|c|c|c|c|c|c|c|c|c|c|c|c|}
\hline $\mathbf{R}$ & 0 & 0 & 0 & 0 & 0 & 0 & 0 & 0 & 0 & 0 & 0 & 0 & 0 & 0 & 0 & 0 & 0 & 66.7 & 0 & 0 \\
\hline $\mathbf{S}$ & 0 & 0 & 0 & 0 & 33.3 & 0 & 0 & 0 & 0 & 0 & 0 & 0 & 0 & 0 & 0 & 0 & 0 & 0 & 100 & 0 \\
\hline $\mathbf{T}$ & 0 & 0 & 0 & 0 & 0 & 0 & 0 & 0 & 0 & 0 & 0 & 0 & 0 & 0 & 0 & 0 & 0 & 0 & 0 & 66.7 \\
\hline Match & $100 \%$ & $100 \%$ & $100 \%$ & $100 \%$ & $66.7 \%$ & $100 \%$ & $33.3 \%$ & $100 \%$ & $100 \%$ & $66.7 \%$ & $33.3 \%$ & $100 \%$ & $100 \%$ & $66.7 \%$ & $100 \%$ & $100 \%$ & $100 \%$ & $66.7 \%$ & $100 \%$ & $66.7 \%$ \\
\hline
\end{tabular}

Table 3 reports confusion matrix generated for True identification of person involved in testing session for PSO with ANN Classifier. It shows the overall True Positive rate of $84.4828 \%$. Where $\mathrm{A}$ to $\mathrm{T}$ in the confusion matrix represents number of clients used in testing the biometric system.

Table 4. Confusion Matrix generated for 20 subjects using PSO+SVM (\%)

\begin{tabular}{|c|c|c|c|c|c|c|c|c|c|c|c|c|c|c|c|c|c|c|c|c|}
\hline & $\mathbf{A}$ & B & $\mathbf{C}$ & D & $\mathbf{E}$ & $\mathbf{F}$ & $\mathbf{G}$ & $\mathbf{H}$ & I & $\mathbf{J}$ & $\mathbf{K}$ & $\mathbf{L}$ & $\mathbf{M}$ & $\mathbf{N}$ & $\mathbf{O}$ & $\mathbf{P}$ & $\mathbf{Q}$ & $\mathbf{R}$ & $\mathbf{S}$ & $\mathbf{T}$ \\
\hline $\mathbf{A}$ & 33.3 & 0 & 0 & 0 & 0 & 0 & 0 & 0 & 0 & 0 & 0 & 0 & 0 & 0 & 0 & 0 & 0 & 0 & 0 & 0 \\
\hline B & 0 & 33.3 & 0 & 0 & 0 & 0 & 0 & 0 & 0 & 0 & 0 & 0 & 0 & 0 & 0 & 0 & 0 & 0 & 0 & 0 \\
\hline C & 0 & 0 & 100 & 0 & 0 & 0 & 0 & 0 & 0 & 0 & 0 & 0 & 0 & 0 & 0 & 0 & 0 & 0 & 0 & 0 \\
\hline $\mathbf{E}$ & 0 & 0 & 0 & 0 & 66.7 & 0 & 0 & 0 & 0 & 0 & 0 & 0 & 0 & 0 & 0 & 0 & 0 & 0 & 0 & 0 \\
\hline $\mathbf{F}$ & 0 & 0 & 0 & 0 & 0 & 33.3 & 0 & 0 & 0 & 0 & 0 & 0 & 0 & 0 & 0 & 0 & 0 & 0 & 0 & 0 \\
\hline $\mathbf{G}$ & 0 & 0 & 0 & 0 & 0 & 0 & 66.7 & 0 & 0 & 0 & 0 & 0 & 0 & 0 & 0 & 0 & 0 & 0 & 0 & 0 \\
\hline H & 0 & 0 & 0 & 0 & 0 & 0 & 0 & 100 & 0 & 1 & 0 & 0 & 0 & 0 & 0 & 0 & 0 & 0 & 0 & 0 \\
\hline I & 0 & 0 & 0 & 0 & 0 & 0 & 0 & 0 & 100 & 0 & 0 & 0 & 0 & 0 & 0 & 0 & 0 & 0 & 0 & 0 \\
\hline $\mathbf{J}$ & 0 & 0 & 0 & 0 & 0 & 0 & 33.3 & 0 & 0 & 100 & 0 & 0 & 0 & 0 & 0 & 0 & 0 & 0 & 0 & 0 \\
\hline $\mathbf{K}$ & 0 & 0 & 0 & 0 & 0 & 0 & 0 & 0 & 0 & 0 & 33.3 & 0 & 0 & 0 & 0 & 0 & 0 & 0 & 0 & 0 \\
\hline $\mathbf{L}$ & 0 & 0 & 0 & 0 & 0 & 0 & 0 & 0 & 0 & 0 & 0 & 100 & 0 & 0 & 0 & 0 & 0 & 0 & 0 & 0 \\
\hline $\mathbf{M}$ & 0 & 0 & 0 & 0 & 0 & 0 & 0 & 0 & 0 & 0 & 0 & 0 & 100 & 0 & 0 & 0 & 0 & 0 & 0 & 0 \\
\hline $\mathbf{N}$ & 66.7 & 0 & 0 & 0 & 0 & 66.7 & 0 & 0 & 0 & 0 & 0 & 0 & 0 & 100 & 0 & 0 & 0 & 0 & 0 & 0 \\
\hline O & 0 & 0 & 0 & 0 & 0 & 0 & 0 & 0 & 0 & 0 & 0 & 0 & 0 & 0 & 100 & 0 & 0 & 0 & 0 & 0 \\
\hline $\mathbf{P}$ & 0 & 0 & 0 & 0 & 0 & 0 & 0 & 0 & 0 & 0 & 0 & 0 & 0 & 0 & 0 & 100 & 0 & 0 & 0 & 0 \\
\hline $\mathbf{Q}$ & 0 & 33.3 & 0 & 0 & 0 & 0 & 0 & 0 & 0 & 0 & 0 & 0 & 0 & 0 & 0 & 0 & 100 & 0 & 0 & 0 \\
\hline $\mathbf{S}$ & 0 & 0 & 0 & 0 & 33.3 & 0 & 0 & 0 & 0 & 0 & 66.7 & 0 & 0 & 0 & 0 & 0 & 0 & 0 & 100 & 0 \\
\hline $\mathbf{T}$ & 0 & 0 & 0 & 0 & 0 & 0 & 0 & 0 & 0 & 0 & 0 & 0 & 0 & 0 & 0 & 0 & 0 & 0 & 0 & 100 \\
\hline Match & $33.3 \%$ & $33.3 \%$ & $100 \%$ & $100 \%$ & $66.7 \%$ & $33.3 \%$ & $66.7 \%$ & $100 \%$ & $100 \%$ & $100 \%$ & $33.3 \%$ & $100 \%$ & $100 \%$ & $100 \%$ & $100 \%$ & $100 \%$ & $100 \%$ & $100 \%$ & $100 \%$ & $100 \%$ \\
\hline
\end{tabular}

Table 5. Confusion Matrix generated for 20 subjects using PSO+K-NN (\%)

\begin{tabular}{|c|c|c|c|c|c|c|c|c|c|c|c|c|c|c|c|c|c|c|c|c|}
\hline & $\mathbf{A}$ & B & C & D & $\mathbf{E}$ & $\mathbf{F}$ & $\mathbf{G}$ & $\mathbf{H}$ & I & $\mathbf{J}$ & $\mathbf{K}$ & $\mathbf{L}$ & $\mathbf{M}$ & $\mathbf{N}$ & $\mathbf{O}$ & $\mathbf{P}$ & $\mathbf{Q}$ & $\mathbf{R}$ & $\mathbf{S}$ & $\mathbf{T}$ \\
\hline $\mathbf{A}$ & 33.3 & 0 & 0 & 0 & 0 & 0 & 0 & 0 & 0 & 0 & 0 & 0 & 0 & 0 & 0 & 0 & 0 & 0 & 0 & 0 \\
\hline B & 0 & 33.3 & 0 & 0 & 0 & 0 & 0 & 0 & 0 & 0 & 0 & 0 & 0 & 0 & 0 & 0 & 0 & 0 & 0 & 0 \\
\hline C & 0 & 0 & 100 & 0 & 0 & 0 & 0 & 0 & 0 & 0 & 0 & 0 & 0 & 0 & 0 & 0 & 0 & 0 & 0 & 0 \\
\hline $\mathbf{E}$ & 0 & 0 & 0 & 0 & 66.7 & 0 & 0 & 0 & 0 & 0 & 0 & 0 & 0 & 0 & 0 & 0 & 0 & 0 & 0 & 0 \\
\hline $\mathbf{F}$ & 0 & 0 & 0 & 0 & 0 & 100 & 0 & 0 & 0 & 0 & 0 & 0 & 0 & 0 & 0 & 0 & 0 & 0 & 0 & 0 \\
\hline $\mathbf{G}$ & 0 & 0 & 0 & 0 & 0 & 0 & 66.7 & 0 & 0 & 0 & 0 & 0 & 0 & 0 & 0 & 0 & 0 & 0 & 0 & 0 \\
\hline H & 0 & 0 & 0 & 0 & 0 & 0 & 0 & 100 & 0 & 1 & 0 & 0 & 0 & 0 & 0 & 0 & 0 & 0 & 0 & 0 \\
\hline I & 0 & 0 & 0 & 0 & 0 & 0 & 0 & 0 & 100 & 0 & 0 & 0 & 0 & 0 & 0 & 0 & 0 & 0 & 0 & 0 \\
\hline $\mathbf{J}$ & 0 & 0 & 0 & 0 & 0 & 0 & 0 & 0 & 0 & 100 & 0 & 0 & 0 & 0 & 0 & 0 & 0 & 0 & 0 & 0 \\
\hline $\mathbf{K}$ & 0 & 0 & 0 & 0 & 0 & 0 & 0 & 0 & 0 & 0 & 100 & 0 & 0 & 0 & 0 & 0 & 0 & 33.3 & 0 & 0 \\
\hline $\mathbf{L}$ & 0 & 0 & 0 & 0 & 0 & 0 & 0 & 0 & 0 & 0 & 0 & 100 & 0 & 0 & 0 & 0 & 0 & 0 & 0 & 0 \\
\hline $\mathbf{M}$ & 0 & 0 & 0 & 0 & 0 & 0 & 0 & 0 & 0 & 0 & 0 & 0 & 100 & 0 & 0 & 0 & 0 & 0 & 0 & 0 \\
\hline $\mathbf{N}$ & 66.7 & 0 & 0 & 0 & 33.3 & 0 & 0 & 0 & 0 & 0 & 0 & 0 & 0 & 100 & 0 & 0 & 0 & 0 & 0 & 0 \\
\hline O & 0 & 0 & 0 & 0 & 0 & 0 & 0 & 0 & 0 & 0 & 0 & 0 & 0 & 0 & 100 & 0 & 0 & 0 & 0 & 0 \\
\hline $\mathbf{P}$ & 0 & 33.3 & 0 & 0 & 0 & 0 & 0 & 0 & 0 & 0 & 0 & 0 & 0 & 0 & 0 & 100 & 0 & 0 & 0 & 0 \\
\hline $\mathbf{Q}$ & 0 & 0 & 0 & 0 & 0 & 0 & 0 & 0 & 0 & 0 & 0 & 0 & 0 & 0 & 0 & 0 & 100 & 0 & 0 & 0 \\
\hline $\mathbf{S}$ & 0 & 0 & 0 & 0 & 0 & 0 & 0 & 0 & 0 & 2 & 0 & 0 & 0 & 0 & 0 & 0 & 0 & 0 & 100 & 0 \\
\hline $\mathbf{T}$ & 0 & 0 & 0 & 0 & 0 & 0 & 33.3 & 0 & 0 & 0 & 0 & 0 & 0 & 0 & 0 & 0 & 0 & 0 & 0 & 100 \\
\hline Match & $33.3 \%$ & $33.3 \%$ & $100 \%$ & $100 \%$ & $66.7 \%$ & $100 \%$ & $66.7 \%$ & $100 \%$ & $100 \%$ & $66.7 \%$ & $100 \%$ & $100 \%$ & $100 \%$ & $100 \%$ & $100 \%$ & $100 \%$ & $100 \%$ & $66.7 \%$ & $100 \%$ & $100 \%$ \\
\hline
\end{tabular}

Table 4 reports confusion matrix obtained for True identification of person involved in testing session for PSO with SVM Classifier. It shows the overall True Positive rate of $82.80 \%$. Table 5 reports confusion matrix of True identification of person involved in testing session for PSO with K-NN Classifier. It shows the overall True Positive rate of $87.9310 \%$. Feature Optimization methods show enhanced results when compared with the features which are directly fed to machine learning classification algorithms. This is evident from the results reported in Table 6. By introducing optimized feature selection it also improves classifier performance indices of accuracy, recall, precision, $\mathrm{F}$ score and specificity. 
Table 6. Performance Parameters

\begin{tabular}{|c|c|c|c|c|c|c|c|c|}
\hline S.No & Techniques & $\begin{array}{c}\text { True_Classifica } \\
\text { tion Rate(TPR) }\end{array}$ & $\begin{array}{c}\text { True_Identificati } \\
\text { on Rate(TPR) }\end{array}$ & $\begin{array}{c}\text { Overall } \\
\text { Classification } \\
\text { Accuracy }\end{array}$ & F Score & Specificity & Recall & Precision \\
\hline 1 & ANN & 70.821 & 80.092 & 91.850 & 73.105 & 95.24 & 75.342 & 77.721 \\
2 & SVM & 73.234 & 81.958 & 93.845 & 77.725 & 96.842 & 77.720 & 83.452 \\
3 & KNN & 72.609 & 81.246 & 92.424 & 76.451 & 95.428 & 76.962 & 80.163 \\
4 & PSO+ANN & 75.615 & 84.482 & 96.448 & 84.321 & 97.183 & 85.00 & 90.976 \\
5 & PSO+SVM & 74.137 & 82.800 & 97.275 & 84.119 & 98.0925 & 83.333 & 91.226 \\
6 & PSO+KNN & 76.847 & 87.931 & 97.893 & 88.892 & 98.3653 & 88.333 & 91.333 \\
\hline
\end{tabular}

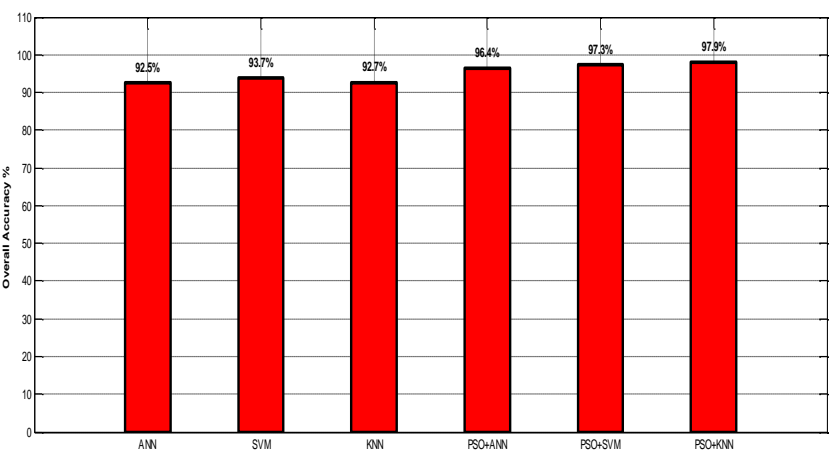

Fig.9 Overall Accuracy Comparison
Fig.9 shows overall accuracy comparison of biometric system using various methodologies. It is noticed that proposed swarm based optimization technique (PSO) shows accurate results when compared to direct classification algorithms. PSO with ANN shows an improvement of $4.598 \%$ when compared to ANN. Similarly PSO with SVM shows an enhancement of $3.430 \%$ when compared to SVM. Further, PSO with K-NN shows an increment of $5.469 \%$, when compared to K-NN. Finally, the proposed PSO+KNN show an accuracy of $97.8931 \%$ for the biometric human recognition system. The proposed methodology is compared with existing literature shown in Table 7.

Table 7. Comparison with previous works

\begin{tabular}{|c|c|c|c|}
\hline Author & Features & Techniques & Accuracy \\
\hline $\begin{array}{l}\text { TS Lugovaya, et al. } \\
2005[24]\end{array}$ & Wavelet Coefficients & $\begin{array}{l}\text { LDA and Majority } \\
\text { Vote Classifier }\end{array}$ & $96 \%$ \\
\hline $\begin{array}{l}\text { Adrian, et al. } \\
2008 \text { [25] }\end{array}$ & $\begin{array}{l}\text { Correlation coefficients from } \\
\text { P-QRS-T Complexes }\end{array}$ & $\begin{array}{l}\text { Percent Residual difference (PRD) } \\
\text { Correlation Coefficient (CC) }\end{array}$ & $\begin{array}{l}70 \%(\mathrm{PRD}) \\
80 \%(\mathrm{CC})\end{array}$ \\
\hline $\begin{array}{l}\text { Boumbarov, et al. } \\
2009 \text { [26] }\end{array}$ & Linear subspace projection & RBFNN & $81.60 \%$ \\
\hline $\begin{array}{l}\text { Shen, et al. } \\
2011[27]\end{array}$ & Non Fiducial & $\mathrm{K}-\mathrm{NN}$ & $95.30 \%$ \\
\hline $\begin{array}{c}\text { Khirul, et al. } \\
2012[28]\end{array}$ & Normalized QRS Complexes & MLPNN & $96.10 \%$ \\
\hline $\begin{array}{l}\text { S Gutta, et al. } \\
\text { 2016 [29] }\end{array}$ & Fiducial Features & $\mathrm{K}-\mathrm{NN}$ & $94.50 \%$ \\
\hline Proposed Study* & $\begin{array}{l}\text { Fiducial based features } \\
\qquad \mathrm{N}=72\end{array}$ & $\begin{array}{c}\text { ANN, SVM, KNN with Particle Swarm } \\
\text { Optimization }\end{array}$ & $\begin{array}{c}97.8931 \% \\
(\mathrm{PSO}+\mathrm{KNN})\end{array}$ \\
\hline
\end{tabular}

\section{CONCLUSION}

In this work, a new effective technique for feature optimization has been proposed. An experimental result shows that the feature optimization technique greatly improves the generalization performance of the classifier for biometric system. In this paper, Particle Swarm Optimization is developed to reduce feature space by removing irrelevant, redundant and over-fitted features based on a fitness function with 
entropy. The proposed method validated by ECG signals

from 20 subjects, each five individual from public available ECG ID database. Performance of proposed biometric recognition using ECG is tested with different classifiers, namely SVM, ANN and KNN with and without optimized feature selection. Finally, the lower number of optimized features gives better identification results when compared to other methods, in which proposed PSO+KNN shows an improvement of $5.4399 \%$ with identification rate of $97.8931 \%$.

\section{REFERENCES}

1. A.Ross, A.K Jain, "Human recognition using biometrics", Annals of Telecommunications, vol.62, pp.11-35, 2007.

2. Irvine M, Israel A and Cheng A, "ECG to Identify Individuals", Pattern recognition, vl.38, pp.133-142, 2005.

3. L Khalil, F.Sufi and J.Hu, "ECG based authentication", Handbook of Information and Communication security Springer , pp.309-331, 2010.

4. C. Ye, M. Coimbra and B. Kumar, "Investigation of human identification using two lead ECG signals" Proceedings of 4th IEEE Int. Conference on Biometrics Theory and Applications (IEEE BTAS), pp.01-08, 2010.

5. B. Nasri, M Guennoun and K. El-Khatib, "Using ECG as a measure in Biometric Identification systems", Proceedings IEEE Toronto Int. Conf. Science and Technology for humanity, pp.28-33, 2009.

6. Tilendra Choudhary, M. Sabarimalai Manikandan "A Novel Unified Framework for Noise-Robust ECG-Based Biometric Authentication" IEEE 2nd International Conference on Signal Processing and Integrated Networks (SPIN), pp.186-191, 2015

7. Sairul Safie, M I Yusof, Kushsairy Kadir, Haidawati Nasir "Bipolar Pulse Active Features for ECG Biometric Application" International Conference on BioSignal Analysis, Processing and Systems (ICBAPS), pp. 01-05, 2015 .

8. Md. Khayrul Bashar, Yuji Ohta, and Hiroaki Yoshida "ECG-based Biometric Authentication Using Mulscale Descriptors" Signal Processing, Computer Networks and Telecommunications, pp.01-04, 2015

9. Abhijit Sarkar, A. Lynn Abbott, Zachary Doerzaph "ECG Biometric Authentication Using a Dynamical Model", 7th IEEE Int. Conf. on Biometrics Theory, Applications and Systems, pp.01-06, 2015.

10. Yue Zhang, Youqun Shi, "A New Method for ECG Biometric Recognition using a Hierarchical Scheme Classifier",6th IEEE International Conference on Software Engineering and Service Sciences,pp.457-460, 2015.

11. Fatema-tuz-Zohra Iqbal, Khairul Azami Sidek, "Cardioid Graph Based ECG Biometric Recognition Incorporating Physiological Variability" Proc. of the IEEE Int. Conf. on Smart Instrumentation, Measurement and Applications , pp.01-05, 2014.

12. Hatzinakos D, Agrafioti F, Gao J, "Heart Biometrics: Theory, methods and applications" Biometrics B , vl.3, pp.199-216, 2011.

13. Wan Y, Yao J, “ A Neural network to identify human subjects with Electrocardiogram signals", World congress on Engineering and Computer Science; 2008, USA

14. Belgcem N, Ali A, Fouenier, "ECG based human authentication using wavelets and random forests", International Journal of Cryptography Information Security, vol.2, pp.01-11, 2012.

15. Kiran Kumar Patro, P. Rajesh Kumar, "A Novel Frequency-Time Based Approach for the Detection of Characteristic Waves in Electrocardiogram Signal”, Springer India , vol.372, no.1, pp.57-67, 2015

16. LugovayaT.S.,"Biometric human identification based on electrocardiogram", [Master's thesis] Faculty of Computing Technologies and Informatics, Electrotechnical University "LETI", Saint-Petersburg, Russian Federation; June 2005.

17. Kiran Kumar Patro, P. Rajesh Kumar, " Effective Feature Extraction of ECG for Biometric Application”, Journal of Procedia, Elsevier, vol.115, pp.296-306, 2017.

18. Khan M, Quadri. "Effects of using filter based feature selection on the performance of Machine learners using different datasets", BVICAM'S
International Journal of Information Technology, vol.5, no.2, pp.597-603, 2013

19. Adejoke B, Omotoyo M. Review of Feature selection Methods in Medical Image Processing, vol.4, no.1, pp.01-05, 2014.

20. Kennedy J. Particle Swarm Optimization, Encyclopedia of Machine Learning, Springer USA: 760-766.

21. I. Defalco, A D Cioppa, E. Tarantino, “ Facing Classification Problems with Particle Swarm Optimization", Applied soft Computing, vol.7, pp.652-658, 2007.

22. $\mathrm{Xu} \mathrm{Y,} \mathrm{Jones} \mathrm{G,} \mathrm{Wang} \mathrm{B.} \mathrm{A} \mathrm{Study} \mathrm{on} \mathrm{Mutual} \mathrm{Information} \mathrm{based} \mathrm{Feature}$ selection for text Categorization, International Journal of Computational Information Systems, vol.3, no.3, pp.1007-1012, 2007.

23. http://www.physionet.org/cgi-bin/atm/ATM - "MIT-BIH ECG ID Database.

24. T.S Lugovaya, AP Nemriko, " Biometric human identification based on Electrocardiogram", Proc. XII-th Russian Conference on Mathematical Methods of Pattern recognition, Moscow 2005:387-390.

25. Adrina, D.C Chan, M Hamdy, "Wavelet distance measure for person identification using electrocardiograms", IEEE transactions on Instrumentation and measurement, vol.57, no.2, pp.248-253, 2008

26. O. Boumbarov, Y.Velchev, S.Sokolov, "ECG Personal identification in subspaces using Radial Basis Nueral Networks", IEEE Int. workshop on Intelligent data acquisition and Advanced Computing systems, pp.446-451, 2009.

27. W D Shen, W J Tompkins, and Y H Hu, "Implementation of a one-lead ECG human identification system on a normal population", Journal of Engg. Comput. Innovations, vol.2, no.1, pp.12-21 ,2011

28. K.Azmi, I Khalil, M Smole, " ECG Biometric Recognition in different physiological conditions using robust normalized QRS' Complexes.cinc.org Computing in Cardiology, vol.39, pp.97-100, 2012.

29. S Gutta, Qi Cheng, " Joint Feature Extraction and Classifier design for ECG based Biometric Recognition”, IEEE Journal of Biomedical and Health Informatics, vol.20, no.2, pp.460-468, 2016.

\section{Authors ProfiLe}

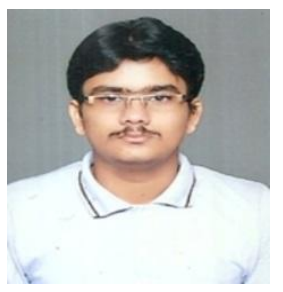

Kiran Kumar Patro received his M.E and Ph.D degree from Department of Electronics and communication Engineering, Andhra University, Visakhapatnam. His research areas include Biomedical signal processing, Image Processing and Machine learning. He is currently working as a Assistant Professor in the department of ECE, Aditya Institute of Technology and Management.

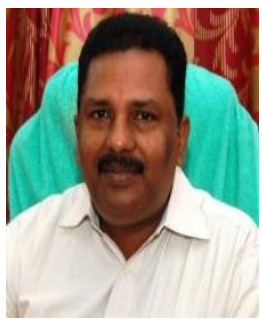

P. Rajesh Kumar is a Professor in Electronics and Communication Engineering department, College of Engineering, Andhra University, Visakhapatnam, India. $\mathrm{He}$ received his M.E. and Ph.D. degree from Andhra University. He has published more than hundred research papers in National and International Journals his research interests are in the area of Digital image processing, Digital signal processing, Radar signal processing and biomedical signal processing

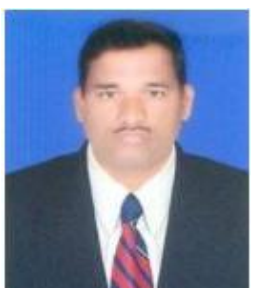

M. Jayamanmadha Rao received his M.Tech and Ph.D degree from Department of Electronics and communication Engineering, Andhra University, Visakhapatnam. His research areas include Image Processing and Signal Processing. He is currently working as a Professor in the department of ECE, Aditya Institute of Technology and Management. 